\title{
Modeling and Measuring Viscoelasticity with Dynamic Atomic Force Microscopy
}

\author{
Per-Anders Thorén, ${ }^{1}$ Riccardo Borgani, ${ }^{1}$ Daniel Forchheimer, ${ }^{1}$ Illia Dobryden, ${ }^{2}$ Per M. Claesson, ${ }^{2}$ \\ Hailu G. Kassa, ${ }^{3}$ Philippe Leclère, ${ }^{3}$ Yifan Wang, ${ }^{4}$ Heinrich M. Jaeger, ${ }^{4}$ and David B. Haviland ${ }^{1, *}$ \\ ${ }^{1}$ Nanostructure Physics, KTH Royal Institute of Technology, Albanova, SE-10791 Stockholm, Sweden \\ ${ }^{2}$ Department of Chemistry, Surface and Corrosion Science, School of Chemical Science and Engineering, KTH \\ Royal Institute of Technology, Drottning Kristinas väg 51, SE-100 44 Stockholm, Sweden \\ ${ }^{3}$ Laboratory for Chemistry of Novel Materials, Center for Innovation and Research in Materials and Polymers, \\ University of Mons, Place du Parc 20, 7000 Mons, Belgium \\ ${ }^{4}$ James Franck Institute, University of Chicago, 929 East 57th Street, Chicago, Illinois 60637, USA
}

(Received 11 October 2017; revised manuscript received 7 May 2018; published 14 August 2018)

\begin{abstract}
The interaction between a rapidly oscillating atomic-force-microscope tip and a soft-material surface is described with use of both elastic and viscous forces in a moving-surface model. We present the simplest form of this model, motivating our derivation with the models ability to capture the impact dynamics of the tip and sample with an interaction consisting of two components: interfacial or surface force, and bulk or volumetric force. Analytic solutions to the piecewise linear model identify characteristic time constants, providing a physical explanation for the hysteresis observed in the measured dynamic-force-quadrature curves. Numerical simulation is used to fit the model to experimental data, and excellent agreement is found with a variety of different samples. The model parameters form a dimensionless impact-rheology factor, giving a quantitative physical number to characterize a viscoelastic surface that does not depend on the tip shape or cantilever frequency.
\end{abstract}

DOI: 10.1103/PhysRevApplied.10.024017

\section{INTRODUCTION}

An increasingly important application of atomic force microscopy (AFM) is the characterization of viscoelastic materials and interfaces, such as cell membranes and tendons [1-4], polymer blends and composites [5-11], liquid-gas and liquid-solid interfaces [12,13], and suspended membranes [14]. The present trend toward higher scanning speeds $[15,16]$ and higher-resolution mapping of mechanical properties $[17,18]$ necessitates more rapid tip motion and therefore a larger viscous contribution to the tip-sample force. Viscous force may even dominate over elastic force with soft materials, and a proper characterization of the material must therefore rely on a dynamic measurement that distinguishes viscous force from elastic force. Here we employ such a method to demonstrate how traditional models for extracting material properties from atomic force microscopy data fail to explain measurements on soft materials. We introduce the simplest form of

*haviland@kth.se

Published by the American Physical Society under the terms of the Creative Commons Attribution 4.0 International license. Further distribution of this work must maintain attribution to the author(s) and the published article's title, journal citation, and DOI. an alternative model that does explain the data. We show how our model parameters relate to physically meaningful numbers that characterize the mechanical response of the viscoelastic surface.

The tip oscillating in and out of contact with the sample in AFM, is a nanometer-scale example of a broader class of dynamic systems known as impact oscillators. These are often modeled with piecewise nonsmooth impact forces that produce interesting bifurcations [19]. The oscillation in dynamic atomic force microscopy typically has a frequency close to a cantilever resonance with high quality factor $Q$, where the inertial and linear restoring forces of the cantilever body dominate the system dynamics. Nevertheless, much smaller impact forces that weakly perturb the harmonic motion of the cantilever can be obtained with the help of lock-in measurement techniques. We use a well-established multifrequency lock-in method where many Fourier components of this nonlinear perturbation are detected as intermodulation products near resonance [20], providing a great deal of information about the impact forces.

When a rigid tip impacts the soft sample it experiences different types of force: elastic force associated with strain in the contact volume and curvature of the contacting interface, and viscous force associated with the rate of change of strain and curvature. These sample deformations occur at the nanometer scale, where the surface-to-volume ratio 
is much larger than that of macroscopic contact mechanics. We therefore expect interfacial forces to play an important role in atomic force microscopy. A viscoelastic model contact forces in AFM must look beyond bulk rheology to also include the rheology of the interface [21].

The traditional approach to quantitative AFM is based on contact mechanics, where the tip-sample force is considered to result from bulk elasticity. The Hertz model [22] gives a repulsive contact force. Assuming an axially symmetric rigid tip, one can parameterize the tip profile in terms of a power $m \in[1,2]$ and a length scale $\ell$ [23] to express the contact force as a function of tip indentation $z_{0}-z$, where $z$ is the position of the tip and $z_{0}$ is the equilibrium position of the surface [see Fig. 1(a)]:

$$
F_{\text {con }}(z)=2 E_{\text {eff }} \alpha_{c}(m) \ell^{2-m}\left(z_{0}-z\right)^{m},
$$

where $E_{\text {eff }}$ is the effective modulus and $\alpha_{c}(m)$ the contact radius correction factor. Typically one does not know $\ell, m$, or $z_{0}$, and they should therefore be treated as free parameters when one is fitting the measured force curve $F_{T S}(z)$ to extract $E_{\text {eff. }}$ With stiff samples one should not assume that the tip is rigid, in which case several more parameters are required to get the material modulus from $E_{\text {eff }}$. Whatever the case, this class of models gives only static force that does not explain dissipation and cannot say anything about the sample viscosity, as it gives rise to a velocity-dependent force.

A viscoelastic version of the Hertz model was studied in the context of two-body collisions, where impact forces can be calculated in the center-of-mass reference frame [24]. With the center of mass in the laboratory frame, defined as the inertial reference frame where the entire sample is at rest (i.e., sample mass much greater than cantilever mass), the viscoelastic Hertz model reduces to a Kelvin-Voigt expression for the tip-sample force:

$$
F_{T S}=F_{\text {con }}(z)+\dot{z} \eta(z),
$$

where dissipation is introduced via a viscous damping coefficient $\eta(z)$ that depends on tip position. One can in principle extract $\eta(z)$ from a dynamic AFM measurement [25], but relating it to sample viscosity would involve a complicated model requiring knowledge of the tip geometry. Other models of contact viscoelasticity use a creepcompliance picture, where the elastic modulus is time dependent and force is determined by integration over deformation history [1-3]. One can also use finite-element methods with linear [26] or nonlinear force-displacement relations that account for an attractive tip-sample force [27].

Independent of these bulk viscoelastic models, tipsample force in AFM may also result from interfacial energy or surface tension $\gamma$. The work of adhesion is the
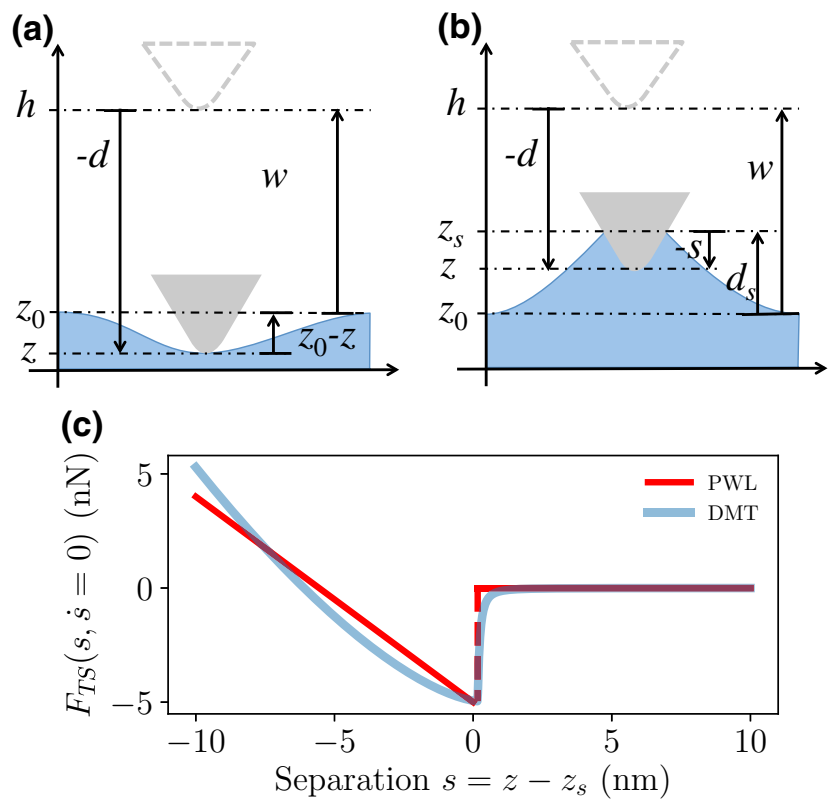

FIG. 1. Coordinates and piecewise linear (PWL) interaction. The cantilever deflection $d=z-h$ is measured by the atomic force microscope detector, where $z(t)$ is the instantaneous tip position and the constant $h$ is the equilibrium (zero force) tip position. $z_{0}$ is the equilibrium position of the sample surface. (a) The traditional view of contact forces in AFM has the tip and surface moving together when they are in contact, $z=z_{s}$, and interaction force is considered to be a function of the surface indentation $\left(z_{0}-z\right)$. (b) The moving-surface model treats the surface position $z_{s}(t)$ as an independent dynamic variable. The model introduces elastic and viscous forces that depend on surface deflection $d_{s}=z_{s}-z_{0}$ and velocity $\dot{d}_{s}$, and an interaction force that depends on the separation $s=z-z_{s}$ and $\dot{s}$. Forces are balanced in the inertial reference frame where the cantilever has a fixed working distance to the sample, $w=h-z_{0}$. (c) The PWL interaction force plotted together with a calculated DerjaguinMuller-Toporov (DMT) model frequently used in AFM [31]. The parameters for the DMT model are as follows: reduced modulus $E^{*}=100 \mathrm{MPa}$, Hamaker constant $H=8 \times 10^{-20} \mathrm{~J}$, tip radius $R=10 \mathrm{~nm}$, intermolecular spacing $a_{0}=0.16 \mathrm{~nm}$. The parameters for the PWL model are as follows: $F_{\text {ad }}=5 \mathrm{nN}, k_{v}=0.9$ $\mathrm{N} / \mathrm{m}$. The range of separation shown is that typically covered by the cantilever oscillation for dynamic AFM on soft materials.

change in total surface energy after contact:

$$
W=\left(\gamma_{T}+\gamma_{S}\right)-\gamma_{T S}
$$

where the subscripts $T$ and $S$ refer to the tip and the sample, respectively. Typically $W>0$, giving an attractive force on contact. The role of surface energy in small mechanical contacts was originally discussed by Johnson, Kendal, and Roberts (JKR) [28]. The JKR model has been shown to break down when the contact radius of curvature $R$ is small in comparison with the elastocapillary length, $L=\Upsilon / E$, 
where $\Upsilon$ is the surface stress and $E$ is the Young's modulus of the sample bulk $[29,30]$. For a soft material with $E \sim 3 \mathrm{MPa}$ forming a contact with relatively low interfacial energy $\gamma_{T S} \sim 30 \mathrm{mN} / \mathrm{m}$ and no additional surface stress (in which case $\Upsilon=\gamma_{T S}$ ), we find $L \sim 10 \mathrm{~nm}$, the typical radius of an AFM tip.

Thus, a tip contacting a soft material should resemble a liquid-like sample wetting and forming a meniscus around the tip, as opposed to the tip compressing an elastic solid. Indeed, $W$ is called the "spreading parameter" in the context of wetting phenomena if the sample corresponds to the liquid and the tip corresponds to the solid substrate, and $W>0$ corresponds to total wetting.

The preceding discussion makes clear that a threedimensional continuum model of impact forces in AFM is difficult to formulate if one includes both bulk and interfacial phenomena, and both elastic and viscous contributions. Knowledge of the tip and sample geometry is required, something that is difficult to determine either before or after an AFM measurement. Even if a realistic model could be formulated, determining its many free parameters from AFM data appears to be a hopeless task because the AFM measurement gives the dynamics of only one degree of freedom; namely, the vertical position of the tip $z(t)$. The formulation of reduced models that approximate the tip-sample collision dynamics is therefore well motivated.

The basic assumption of nearly all models in AFM is that the tip-sample interaction force can be expressed as a function of two dynamic variables:

$$
F_{T S}(t)=f(z(t), \dot{z}(t)) .
$$

We argue that this basic assumption is incorrect for soft materials as it ignores the fact that the sample itself also has dynamics, which clearly influence the tip-sample interaction. Our approach is to reduce the complicated three-dimensional dynamics of the sample to a simplified one-dimensional dynamics in terms of a single degree of freedom $z_{s}(t)$, a generalized or spatially averaged vertical position of the surface in the laboratory frame [see Fig. 1(b)]. If we disregard the inertia of the sample contained in the very small interaction volume, our interaction force depends on this one additional "hidden" dynamic variable:

$$
F_{T S}(t)=f\left(z(t), \dot{z}(t), z_{S}(t)\right) .
$$

This type of model was introduced by Cantrell and Cantrell [32] to account for externally forced oscillations of the sample in the context of ultrasonic AFM.

In the following sections we describe a simplified version of the moving-surface model. A more complicated variation was presented and compared with data from experiments on soft materials in our previous publication [33]. The simpler model has analytic solutions in special cases that give physical intuition. We simulate the simplified model and introduce numerical optimization to find the model parameters that best fit the experimental data. We argue that the traditional approach to quantitative AFM, where model parameters are bulk material properties such as elastic modulus and viscosity, is not appropriate for soft materials because a physically correct and complete model would involve far too many free parameters and uncontrolled assumptions. Rather, we demonstrate that a dimensionless ratio of our model parameters, called the "impact-rheology factor" $R$, gives a physically relevant and useful quantity for nanoscale mechanical characterization of viscoelastic surfaces. Our measurements show that the impact-rheology factor is independent of the oscillation frequency and the tip geometry.

\section{THE MOVING-SURFACE MODEL}

All coordinates of the moving-surface model are defined in Fig. 1(b). The essential difference from the traditional approach [Fig. 1(a)] is that we introduce the instantaneous position of the surface $z_{s}(t)$ as an independent dynamic variable and we express the interaction force as a function of the tip-surface separation $s=z-z_{s}$. Unlike traditional models, we do not require that the tip and surface move together (i.e., $z=z_{s}$ ) when they are in contact.

One could imagine many different nonlinear models for the interaction force, but we make a sweeping simplification by linearizing the interaction when there is contact. Figure 1(c) shows a piecewise linear (PWL) force model described by the following equations:

$$
F_{T S}(s, \dot{s})= \begin{cases}0 & \text { if } s>0 \\ -F_{\mathrm{ad}}-k_{v} s-\eta_{v} \dot{s} & \text { if } s \leq 0\end{cases}
$$

The interaction force is zero when the tip is out of contact, $s>0$. When the tip is in contact, $s \leq 0$, the adhesion force $F_{\text {ad }}$ turns on, corresponding to a lowering of the total interfacial energy. Adhesion is counteracted by a repulsive force linear in the penetration $-s$, with force constant $k_{v}$. We also include the possibility of a viscous force, linear in $\dot{s}=\dot{d}-\dot{d}_{s}\left(h\right.$ and $z_{0}$ are constant), associated with material flow as the tip penetrates the sample.

The model preserves one essential feature of the interaction that is well known in AFM: the large force gradient $(d / d z) F_{T S}(s, \dot{s})$ localized near the point of contact $s=0$. This rapid change of force is responsible for the jump-tocontact and pull-off hysteresis seen in nearly all quasistatic force curves, or measurements of $d(h)$ for small $\dot{h}$, traditionally analyzed in quasistatic AFM. In dynamic AFM the amplitude of oscillation is typically much larger than the range of this localized interaction. We may therefore approximate this region of large interaction gradient as an adhesion force that instantly turns on and off when crossing the point of contact $s=0$. 
The interaction force couples the dynamics of the cantilever's flexural eigenmode to the dynamics of the viscoelastic surface, described by the following set of equations:

$$
\begin{gathered}
m \ddot{d}+\eta \dot{d}+k d=F_{T S}(s, \dot{s})+F_{\text {drive }}(t), \\
\eta_{s} \dot{d}_{s}+k_{S} d_{s}=-F_{T S}(s, \dot{s}),
\end{gathered}
$$

where $d_{s}=z_{s}-z_{0}$ is the deflection of the surface from its equilibrium position $z_{0}$ and $F_{\text {drive }}(t)$ is the drive force.

The free motion of the tip is given by Eq. (7a) with $F_{T S}=0$, describing a driven damped harmonic oscillator. This model is valid in a narrow frequency band around a high- $Q$ resonance of the cantilever. The three mode parameters - stiffness $k$, resonant frequency $\omega_{0}=$ $2 \pi f_{0}=\sqrt{k / m}$, and dimensionless quality factor $Q=$ $\omega_{0} \tau=\sqrt{\eta^{2} / m k}$ - are independently determined by a calibration procedure (see Appendix A).

The free motion of the surface is described by Eq. (7b) with $F_{T S}=0$. Note that Eq. (7b) does not have an inertial term corresponding to force arising from acceleration of the sample mass. Disregarding sample mass is valid when deformation occurs only in a local volume around the tip; however, inertial forces may arise if the cantilever excites surface waves [33]. Thus, the model effectively puts the center of mass at the equilibrium position of the tip, which we treat as fixed in the laboratory frame. We therefore disregard the very small amplitude base motion needed to inertially actuate the high- $Q$ resonance [25], as well as any forces arising from rapid changes of the probe height due to overly active surface-tracking feedback.

We can develop intuition for the sample dynamics by considering what happens when the tip is held rigidly fixed in the laboratory frame (i.e., not connected to a flexible cantilever) at the height of the unperturbed surface. As the tip just touches the surface from above [see Fig. 2(a)], the case $s=0$ in Eq. (6) together with the condition $z=z_{0}$, or equivalently $s=-d_{s}$ in Eq. (7b), gives

$$
\left(\eta_{s}+\eta_{v}\right) \dot{d}_{s}+\left(k_{s}+k_{v}\right) d_{s}=F_{\mathrm{ad}} .
$$

Solving this equation, we find that on contact the adhesion force lifts the surface,

$$
d_{S}(t)=\delta\left(1-e^{-t / \tau_{c}}\right),
$$

forming a meniscus with asymptotic height

$$
\delta=F_{\text {ad }} /\left(k_{v}+k_{s}\right)
$$

in a characteristic contact formation time

$$
\tau_{c}=\frac{\eta_{v}+\eta_{s}}{k_{v}+k_{s}}
$$

(a)
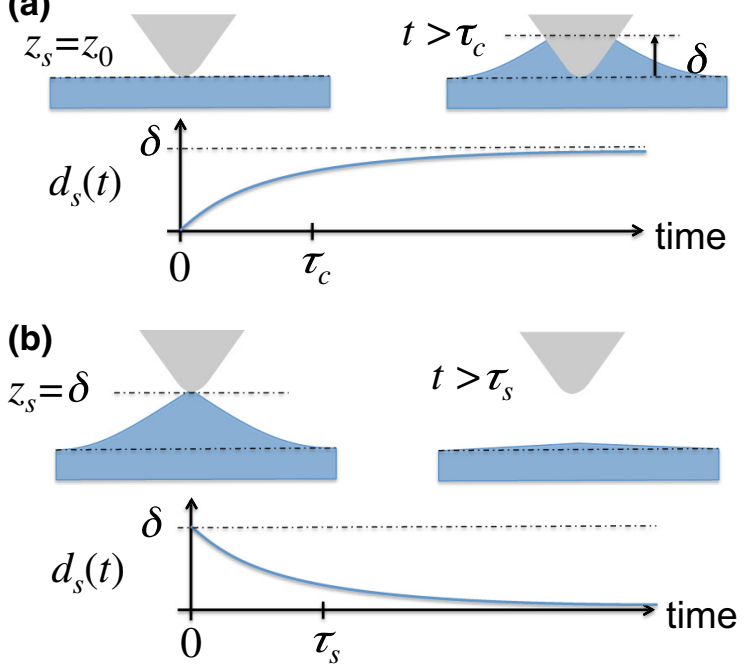

FIG. 2. Contact formation and free relaxation. (a) At $t=0$ a tip, rigidly fixed in the laboratory frame, meets the sample surface. The adhesion force turns on, and the surface lifts by an amount $\delta$, forming the contact in a characteristic time $\tau_{c}$. (b) At $t=0$ the tip separates from the lifted surface. The surface relaxes to its equilibrium position in the characteristic time $\tau_{s}$.

Similarly, when the tip just separates from the lifted surface, Eq. (6) for the case $s>0$ and Eq. (7b) give

$$
\eta_{s} \dot{d}_{s}+k_{s} d_{s}=0
$$

describing free relaxation of the surface to its equilibrium position in a characteristic time

$$
\tau_{s}=\eta_{s} / k_{s}
$$

The contact formation and free relaxation dynamics are depicted in Fig. 2. We may also define the time constant

$$
\tau_{v}=\frac{\eta_{v}}{k_{v}}
$$

associated with tip penetration into the sample.

The model behaves as we would intuitively expect for an interaction consisting of two opposing forces: attractive surface force resulting from minimization of interfacial energy, and repulsive volumetric force resulting from compressive stress in the bulk. The stiffness parameters $k_{s}$ and $k_{v}$ include both surface and bulk forces, with the relative contribution depending on the size of the deformation in relation to the elastocapillary length. Nevertheless, a liquid-like interaction is described by $K \equiv k_{s} / k_{v} \gg 1$, in which case the meniscus lifts to a maximum height

$$
\delta \simeq \delta_{0} \equiv F_{\mathrm{ad}} / k_{s} .
$$

A solid-like interaction is described by the opposite limit $K \ll 1$, in which case $\delta \ll \delta_{0}$ as capillary adhesion is counteracted by compressive stress in the contact volume. 
The time constants $\tau_{c}, \tau_{s}$, and $\tau_{v}$ represent ratios of viscous to elastic force constants of the model. The dynamics of both the tip and the sample depend on these characteristic time constants and their relation to the time spent in and out of contact during a single oscillation cycle, where the latter is set by the frequency of cantilever oscillation, the amplitude of motion, and the working distance to the surface $w=h-z_{0}$. When the contact formation time $\tau_{c}$ is longer than the time spent in contact, the surface cannot fully deform to achieve contact equilibrium. When the free relaxation time $\tau_{s}$ is long compared with the time spent out of contact, the surface cannot relax to its equilibrium position before the next tap of the tip. Repeated taps result in a steady-state dynamics characterized by a time-average uplifted or indented position of the surface.

In the following section we demonstrate this behavior of the model and correlate it with data from experiments. We show that this simple model explains the experimental data remarkably well for a variety of different soft samples. Fitting the model to experimental data, we extract the parameters that characterize the material and its surface. We then comment on how these parameters may be useful for a general, quantitative characterization of viscoelastic samples with AFM.

\section{COMPARISON WITH EXPERIMENT}

We validated the model by fitting it to data collected from several different samples. Figure 3 shows scanned images of four samples we discuss later. To display the results of both experiment and theory, we show dynamic-force-quadrature curves obtained with a a technique called "intermodulation atomic fore microscopy" (ImAFM) [20]. Measurement details are given in Appendix B. Force quadratures were introduced in previous publications $[34,35]$ but they are rather unfamiliar to most scientists who use AFM so we briefly describe them here. Force-quadrature curves do not display the instantaneous force on the tip as a function of tip position $F_{T S}(z)$ (i.e., "force curves"). Rather, force quadratures represent integrals of force over a single oscillation cycle, making them the natural force curves of dynamic AFM.

The reader familiar with dynamic mechanical analysis will recognize a similarity to force quadratures. In dynamic mechanical analysis, the strain in a bulk material is measured while a sinusoidal stress of fixed amplitude is applied. The in-phase and quadrature components of the strain are then monitored as a function of the frequency of excitation (or the temperature of the sample) to characterize the complex modulus $E^{*}=E^{\prime}+i E^{\prime \prime}$ of the material under test. To determine AFM force quadratures, we instead monitor the in-phase and quadrature response of the cantilever, subject to a sinusoidal excitation at fixed
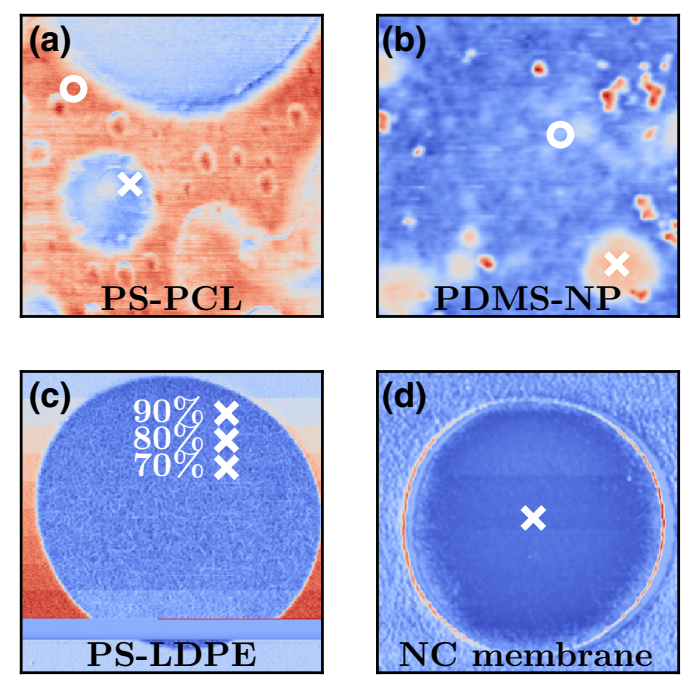

FIG. 3. Phase images at the first drive frequency of intermodulation AFM. (a) Scan size $0.8 \mu \mathrm{m}$. Domains of polycaprolactone (PCL; blue) in polystyrene (PS; red). Pixels marked with $\mathbf{o}$ and $\times$ are analyzed in Fig. 4. (b) Scan size $1 \mu \mathrm{m}$. Polydimethylsiloxane (PDMS; blue) with clusters of hydrophobic silica nanoparticles (NP; red). Pixels marked with $\mathbf{o}$ and $\times$ are analyzed in Fig. 5. (c) Scan size $1.5 \mu \mathrm{m}$. A set-point study on a domain of low-density polyethylene (LDPE; blue) in PS. Pixels marked with $\times$ are analyzed in Figs. 6 and 8 at the set-point values shown. (d) Scan size $3 \mu \mathrm{m}$. A suspended membrane of Au nanoparticles bound together in a monolayer by organic ligands. A pixel at the center of the membrane marked with $\times$ is analyzed in Fig. 7 .

frequency but with slowly varying amplitude. This allows us to study how the nonlinear features associated with the impact of the tip on the viscoelastic sample change with amplitude. To explain these features we must look beyond the bulk concepts of storage and loss moduli to include also surface forces and adhesion.

The high quality factor of the cantilever resonance means that the energy stored in the cantilever oscillation is much larger than the tip-sample interaction potential or energy lost to dissipation in the cycle. The tip motion is therefore well described by harmonic oscillation at a fixed "carrier" frequency $\bar{\omega} \simeq \omega_{0}$. The second drive tone of ImAFM gives rise to a slowly modulated amplitude and phase of the carrier. From the intermodulation spectrum of these two drive tones, which is concentrated near resonance, we extract the Fourier coefficients of the tip motion and tip-sample force at this carrier frequency. Rotating the force coefficients by the motion phase, we project out the Fourier coefficients of force that are in phase with the motion $F_{I}$, and those that are in phase with the velocity $F_{Q}[34,35]$. Both are presented as functions of oscillation amplitude $A$ :

$$
z(t)=h+A \cos \bar{\omega} t,
$$


Sample: PS-PCL
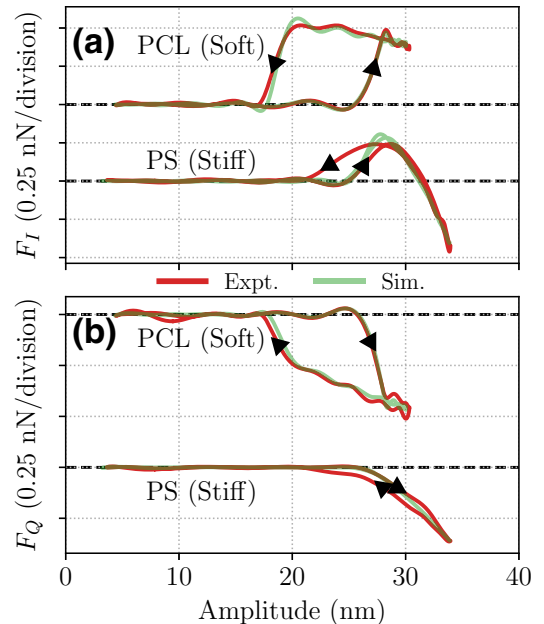

Cantilever: Tap300 $\left(f_{0}=310.6 \mathrm{kHz}, k=26.03 \mathrm{~N} / \mathrm{m}, Q=465.1\right)$
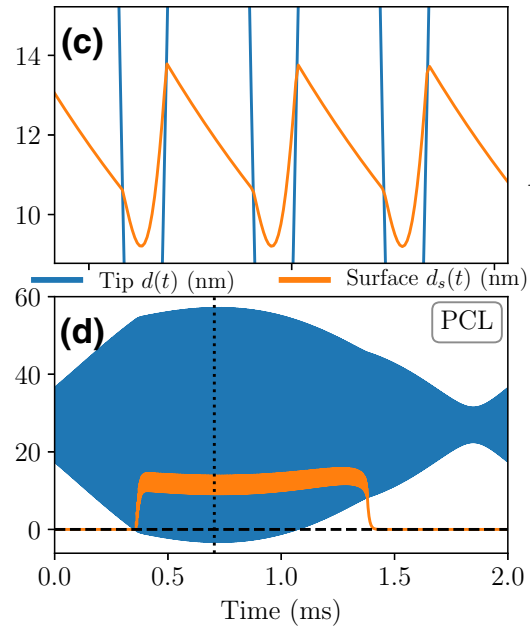
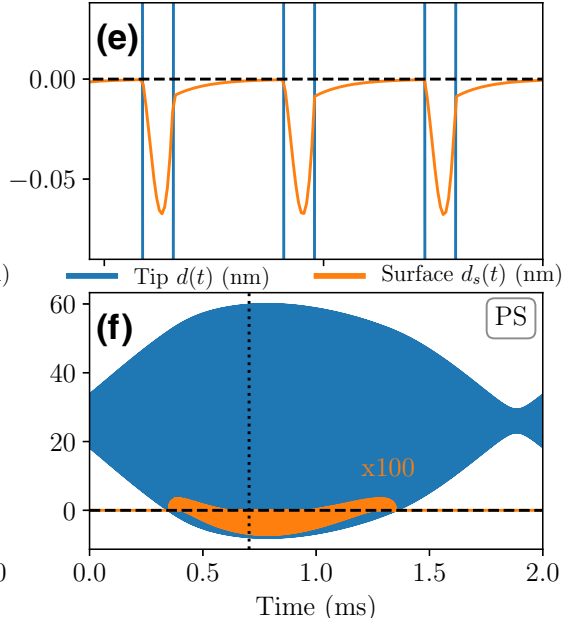

\begin{tabular}{|c|c|c|c|c|c|c|c|c|}
\hline & $h(\mathrm{~nm})$ & $\tau_{\boldsymbol{s}}(\mathrm{ns})$ & $\tau_{\boldsymbol{v}}(\mathrm{ns})$ & $k_{\boldsymbol{S}}(\mathrm{N} / \mathrm{m})$ & $k_{\boldsymbol{v}}(\mathrm{N} / \mathrm{m})$ & $F_{\text {ad }}(\mathrm{nN})$ & $K=k_{\boldsymbol{S}} / k_{\mathrm{v}}$ & $R=\tau_{s} / \tau_{\boldsymbol{v}}$ \\
\hline PCL & 26.95 & 8118 & 580.3 & 0.0469 & 0.1557 & 2.859 & 0.3012 & 13.99 \\
\hline PS & 25.98 & 735.2 & 152.8 & 32.53 & 1.097 & 3.88 & 29.65 & 4.811 \\
\hline
\end{tabular}

FIG. 4. Polystyrene (PS) and polycaprolactone (PCL) blend. (a) The conservative force quadrature $F_{I}(A)$ and (b) the dissipative quadrature $F_{Q}(A)$. The curves are offset vertically for clarity, with dashed lines corresponding to zero force. The simulated motion of the tip $z(t)$ (blue) and surface $z_{s}(t)$ (orange) is shown for both PCL (c),(d) and PS (e),(f). The enlargements in (c),(e) show details of the surface motion around the time marked by the vertical dotted line in (d),(f), respectively. The surface motion of PS in (f) is magnified by a factor of 100. Note that the average surface position for PCL is approximately $12 \mathrm{~nm}$ above its rest position, while PS deviates by only approximately $0.05 \mathrm{~nm}$ from its equilibrium position. The best-fit parameters used in the simulation are given in the table.

$$
\begin{aligned}
& F_{I}(A)=\frac{1}{T} \int_{0}^{T} F_{T S}(t) \cos (\bar{\omega} t) d t, \\
& F_{Q}(A)=\frac{1}{T} \int_{0}^{T} F_{T S}(t) \sin (\bar{\omega} t) d t .
\end{aligned}
$$

$F_{I}$ represents a conservative force (i.e., energy recovered in the oscillation cycle), and $F_{Q}$ represents a dissipative force (i.e., energy lost in the cycle).

When the tip is tapping on the sample, large Fourier components of the tip-sample force also exist at frequencies far above resonance but well below the detection noise floor. Thus, we cannot determine the instantaneous force at any given time in the cycle. However, using the multifrequency lock-in technique embodied in ImAFM, we can determine the integrated force over single cycles with very high signal-to-noise ratio. The two curves $F_{I}(A)$ and $F_{Q}(A)$ are found by direct transformation of the intermodulation spectrum, requiring only the calibrated linear response function of the cantilever eigenmode. No assumptions are made regarding the specific nature of the tip-sample interaction. The transformation is quite efficient computationally, allowing immediate examination of the forcequadrature curves at individual pixels while scanning.

Figure 4 shows force-quadrature curves obtained at two different points on a blend of polystyrene (PS) and polycaprolactone (PCL), shown in Fig. 3(a). The force quadratures on the softer PCL (nominal bulk $E \sim 300$
MPa) show a larger magnitude of the dissipative force $F_{Q}$ and a conservative force that is dominantly attractive, $F_{I}>0$, even at the highest amplitude. To fit the theory to the experimental data, we simulate the model dynamics by numerical integration of the equations of motion [Eqs. (7a) and (7b)], adjusting the parameters of the model to find the best fit. Details of this procedure are given in Appendix C.

On the soft PCL we find an excellent fit. The simulation accurately reproduces the magnitude, complex shape, and hysteresis of both $F_{I}(A)$ and $F_{Q}(A)$. The simulated surface motion shows that the adhesive force causes the surface to lift by a time-averaged distance of about $15 \mathrm{~nm}$ above its equilibrium position [Fig. 4(d)]. The enlargement of individual oscillation cycles [Fig. 4(c)] shows that on impact the tip penetrates as much as approximately $20 \mathrm{~nm}$ below the lifted surface. After separation on the upward trajectory of the tip, the surface does not have time to fully relax to its equilibrium position before the next impact. This slow relaxation of the surface gives rise to the hysteresis observed in the $F_{I}(A)$ and $F_{Q}(A)$ curves: the amplitude at which the surface is initially lifted up [sharp upturn in $\left.F_{I}(A)\right]$ is larger than the amplitude where the oscillating tip fully releases the surface [sharp downturn in $F_{I}(A)$ ]. This hysteresis is not easily explained by a model having the form of Eq. (4), giving confidence that the basic physical picture embodied in the moving-surface model is correct. 
Sample: PDMS with nanoparticles

Cantilever: Tap300 ( $\left.f_{0}=313.9 \mathrm{kHz}, k=22.2 \mathrm{~N} / \mathrm{m}, Q=390.4\right)$
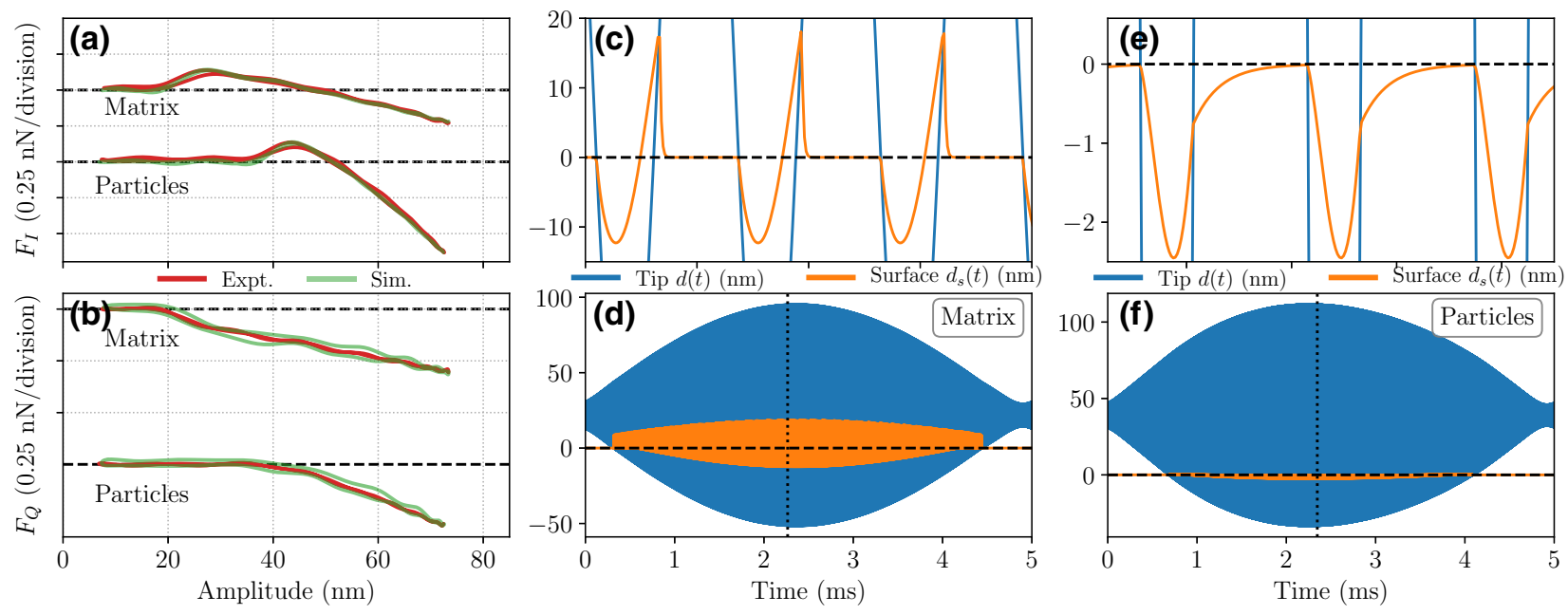

\begin{tabular}{|c|c|c|c|c|c|c|c|c|}
\hline & $h(\mathrm{~nm})$ & $\tau_{\boldsymbol{s}}(\mathrm{ns})$ & $\tau_{\mathbf{v}}(\mathrm{ns})$ & $k_{s}(\mathrm{~N} / \mathrm{m})$ & $k_{\boldsymbol{V}}(\mathrm{N} / \mathrm{m})$ & $F_{\text {ad }}(\mathrm{nN})$ & $K=k_{s} / k_{\boldsymbol{V}}$ & $R=\tau_{s} / \tau_{\boldsymbol{v}}$ \\
\hline Matrix & 21.84 & 31.89 & 398.1 & 0.1326 & 0.0444 & 0.8459 & 2.986 & 0.0801 \\
\hline Particles & 39.08 & 506.5 & 142.5 & 1.067 & 0.1729 & 1.415 & 6.171 & 3.555 \\
\hline
\end{tabular}

FIG. 5. Polydimethylsiloxane (PDMS) with hydrophobic silica nanoparticles. The details of this sample are described in a previous publication [36]. The curves labeled "Matrix" are obtained with the softer PDMS, where the simulation shows large-amplitude surface motion and deep tip penetration. The curves labeled "Particles" are obtained with a subsurface aggregation of nanoparticles, where the simulation reveals very little surface motion and lifting, yet equally deep tip penetration. (a) The conservative force quadrature $F_{I}(A)$ and (b) the dissipative quadrature $F_{Q}(A)$. The curves are offset vertically for clarity, with dashed lines corresponding to zero force. The simulated motion of the tip $z(t)$ (blue) and surface $z_{S}(t)$ (orange) is shown for both the PDMS matrix (c),(d) and a cluster of particles (e),(f). The enlargements in (c),(e) show details of the surface motion around the time marked by the vertical dotted line in (d),(f), respectively. The best-fit parameters used in the simulation are given in the table.

We compare the behavior on the softer PCL with that on the stiffer PS domain (expected $E \sim 3 \mathrm{GPa}$ ). On PS we find that the conservative force quadrature $F_{I}$ becomes dominantly repulsive $\left(F_{I}<0\right)$ at large amplitude, and we see a smaller magnitude of the dissipative force $F_{Q}$. Simulations show that the tip penetrates as much as $10 \mathrm{~nm}$ below the surface, but the surface lifts only slightly and quickly relaxes to its equilibrium position before the next impact. With increasing $A$ the simulation captures the shape and magnitude of both $F_{I}(A)$ and $F_{Q}(A)$. However, with decreasing $A$ we find that the simulation does not reproduce the hysteresis observed in the experiment. A different interaction function $F_{T S}(s)$ could improve the quality of the fit, but at the expense of introducing additional model parameters. In our experience, the simplified model presented herein often does not capture hysteresis observed together with $F_{I}<0$, but it nearly always captures hysteresis when $F_{I}>0$.

Figure 5 shows an additional example of model fitting at two locations on a sample consisting of polydimethylsiloxane (PDMS) mixed with hydrophobic silica nanoparticles, shown in Fig. 3(b). This soft sample has faster free relaxation (smaller $\tau_{s}$ ) such that no hysteresis is seen in the force quadratures. The model reveals large-amplitude surface motion when the tip is tapping on the PDMS matrix.
When the tip is tapping on a region with dispersed nanoparticles, the model shows a much slower surface motion and very low amplitude surface motion, but equally deep penetration.

We also investigate how the fitted model parameters depend on a change of tip and cantilever oscillation frequency. Using three standard cantilevers with calibrated parameters given in Table I, we study a well-known soft material consisting of micron-size domains of low-density polyethylene (nominal bulk $E \sim 100 \mathrm{MPa}$ ) in a matrix of PS [37], as shown in Fig. 3(c). The cantilever resonant frequencies and mode stiffnesses span 1 order of magnitude. To make a reasonable comparison we adjust the excitation to keep the amplitude of free motion $A_{\text {free }}$ such that the energy stored in the free oscillation $E_{\text {free }}=\frac{1}{2} k A_{\text {free }}^{2}$ is approximately the same in each measurement. Table I also shows the best-fit parameters of the moving-surface model.

For each cantilever we also study how the forcequadrature curves change as we vary the working distance, $w=h-z_{0}$, or static probe height above the relaxed surface. We control $w$ by simply changing the scanning feedback set point at regular intervals along the slow scan axis [see Fig. 3(c)]. Figure 6 shows an example of the measured and simulated force quadratures for the Tap525 cantilever at three different probe heights. For all simulation curves 
TABLE I. Parameters for low-density polyethylene obtained with three different cantilevers (NSC15, Tap525, and AC55): $f_{0}$, $Q, k$, and $A_{\text {free }}$ are the calibrated cantilever parameters, $F_{\text {ad }}, k_{s} \eta_{s}$, $k_{v}$, and $\eta_{v}$ are the fitted model parameters, and $\tau_{s}=\eta_{s} / k_{s}$ and $\tau_{v}=\eta_{v} / k_{v}$ (relaxation times), $\tau_{c}=\left(\eta_{s}+\eta_{v}\right) /\left(k_{s}+k_{v}\right)$ (contact formation time), $K=k_{s} / k_{v}$ (stiffness ratio), and $R=\tau_{s} / \tau_{v}$ (impact-rheology factor) are ratios of the model parameters. Although the stiffness and damping parameters of the model show considerable variation between probes with different frequency and tip geometry, the time constants show much less variation. The impact-rheology factor $R$ shows little variation between probes.

\begin{tabular}{lccc}
\hline \hline & NSC15 & Tap525 & AC55 \\
\hline$f_{0}(\mathrm{kHz})$ & 230 & 489 & 2120 \\
$Q$ & 397 & 547 & 735 \\
$k(\mathrm{~N} / \mathrm{m})$ & 18.9 & 88.6 & 161 \\
$A_{\text {free }}(\mathrm{nm})$ & 20 & 11 & 6.3 \\
\hline$F_{\mathrm{ad}}(\mathrm{nN})$ & 0.955 & 1.8 & 0.818 \\
$k_{s}(\mathrm{~N} / \mathrm{m})$ & 0.17 & 0.858 & 0.227 \\
$\eta_{s}(\mathrm{~kg} / \mathrm{s})$ & $3.01 \times 10^{-6}$ & $14.9 \times 10^{-6}$ & $2.75 \times 10^{-6}$ \\
$k_{v}(\mathrm{~N} / \mathrm{m})$ & 0.04 & 0.0238 & 0.014 \\
$\eta_{v}(\mathrm{~kg} / \mathrm{s})$ & $8.24 \times 10^{-8}$ & $4.74 \times 10^{-8}$ & $1.92 \times 10^{-8}$ \\
\hline$\tau_{s}(\mu \mathrm{s})$ & 17.68 & 17.31 & 12.11 \\
$\tau_{v}(\mu \mathrm{s})$ & 2.06 & 1.99 & 1.37 \\
$\tau_{c}(\mu \mathrm{s})$ & 14.7 & 17.1 & 11.5 \\
$K$ & 4.25 & 3.6 & 16.21 \\
$R$ & 8.61 & 8.69 & 8.86 \\
\hline \hline
\end{tabular}

shown in Fig. 6 the same parameters given in Table I are used, with only the working distance $w$ being changed. Equivalent plots for the NSC15 and AC55 cantilevers are shown in Appendix D. We see that the model reproduces the shape and offset of the force-quadrature curves rather well.

To further test the model we also analyze a nanoparticle membrane sample, shown in Fig. 3(d). This sample consists of a monolayer of Au nanoparticles, bound together by organic ligands. The membrane is suspended over a circular hole $2.5 \mu \mathrm{m}$ in diameter, forming an ultrathin drum head $[38,39]$. Studies have shown large apparent stiffness, corresponding to a large effective bulk modulus of the membrane material [40]. Interpretation of the model parameter $k_{v}$ in terms of bulk compression is not obvious with these samples that have thickness of approximately $7 \mathrm{~nm}$. However, adhesion gives rise to very large curvature of the upper and lower surfaces of the membrane as it covers the tip with very small radius. The Laplace pressure change across both of these interfaces adds, giving the force described by $k_{v}$ that acts in opposition to adhesion. The stiffness constant $k_{s}$ then describes elastic tension in the membrane as the surface deviates from equilibrium. It is therefore not surprising that our simple model fits the data quite well. Figure 7 shows a comparison of experimental data and simulation of the model for two different cantilevers. The qualitative shape of the force quadratures
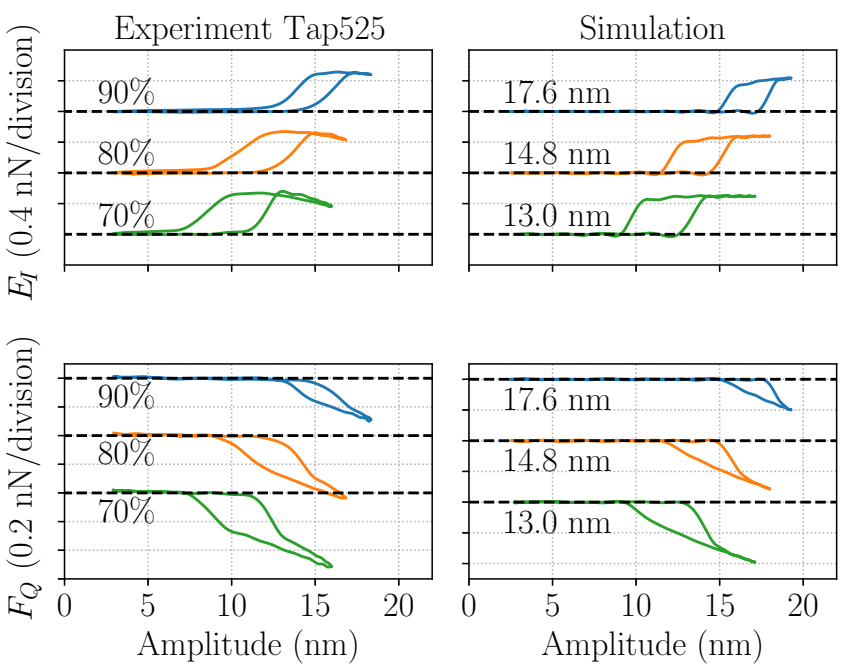

FIG. 6. Low-density polyethylene measured with a Tap525 cantilever. Experimental (left) and simulated (right) forcequadrature curves, each offset vertically for clarity, with dashed lines corresponding to zero force. The experimental curves result from analysis of data at pixels marked with a cross of the corresponding color in Fig. 3(c). The working distance is changed in the experiment by adjustment of the amplitude-feedback set point to the value given in the left panels (percentage of free amplitude). For simulation the working distance $w$ shown in the right panels is found by numerical optimization with all other parameters of the model fixed at the values given in Table I.

and the simulated dynamics of the surface are the same for both cantilevers, despite the difference in the resonance frequencies of the AC55 cantilever (approximately $2 \mathrm{MHz}$ ) and the Tap300 cantilever (approximately $300 \mathrm{kHz}$ ).

\section{DISCUSSION}

An obvious criticism of the model presented herein is that it is ad hoc, or not based on first principles. Nonlinear forces arising from sample deformation, the analysis of which forms the basis of traditional AFM nanomechanics, are avoided by linear approximation. The model keeps only one nonlinearity to capture the sudden impact and release of the tip and sample. Reduction of the sample dynamics to one effective degree of freedom $d_{s}(t)$ is a sweeping simplification that ignores the fact that the surface deformation has a transverse profile.

We emphasize again that our intent is to describe the measured dynamics of the cantilever with a minimum number of free parameters and additional degrees of freedom. A physically more realistic short-range interaction force that smoothly interpolates between the two linear regions could be constructed, but at the expense of additional parameters that are not necessary to explain the data. We consider this to be the minimal model necessary for explaining dynamic AFM on viscoelastic materials, and in our experience it is often sufficient. 
AC55

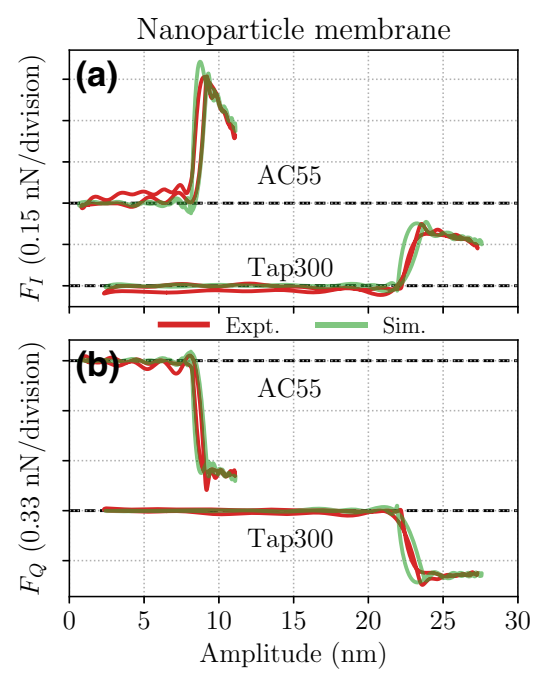

$f_{0}=1.97 \mathrm{MHz}, k=188 \mathrm{~N} / \mathrm{m}, Q=703$
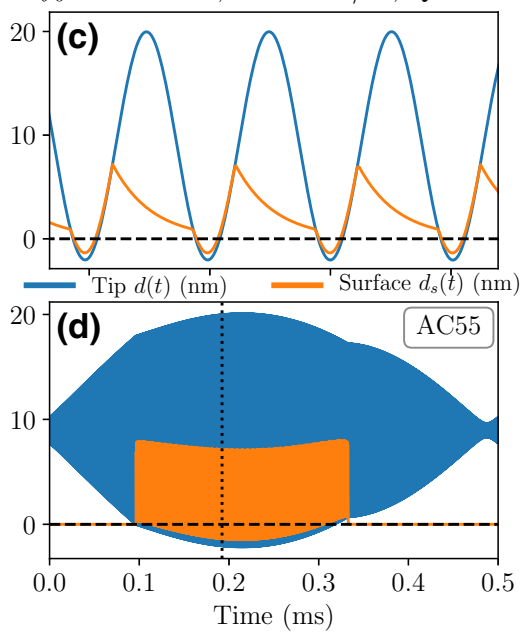

Tap300

$f_{0}=319 \mathrm{kHz}, k=31.9 \mathrm{~N} / \mathrm{m}, Q=549$
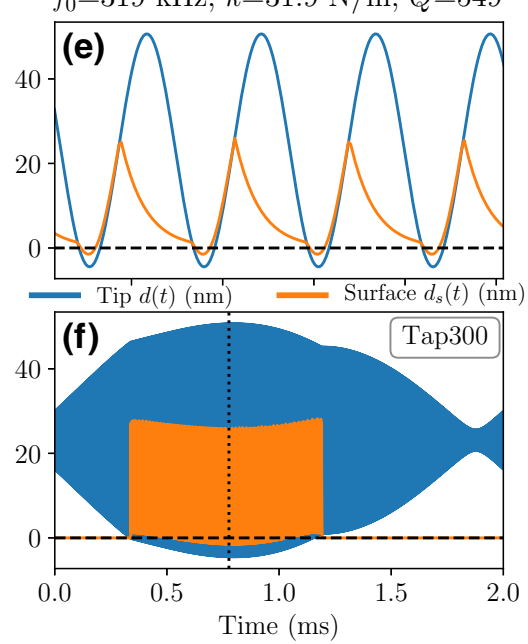

\begin{tabular}{|c|c|c|c|c|c|c|c|c|}
\hline & $h(\mathrm{~nm})$ & $\tau_{S}(\mathrm{~ns})$ & $\tau_{\boldsymbol{V}}(\mathrm{ns})$ & $k_{\boldsymbol{S}}(\mathrm{N} / \mathrm{m})$ & $k_{V}(\mathrm{~N} / \mathrm{m})$ & $F_{\text {ad }}(\mathrm{nN})$ & $K=k_{S} / k_{V}$ & $R=\tau_{s} / \tau_{\boldsymbol{V}}$ \\
\hline AC55 & 8.969 & 1000 & 336.3 & 0.2829 & 6.144 & 4.665 & 0.04605 & 2.974 \\
\hline Tap300 & 23.11 & 663.1 & 239.7 & 0.0597 & 0.7319 & 2.657 & 0.08157 & 2.766 \\
\hline
\end{tabular}

FIG. 7. Nanoparticle membrane measured with Tap300 and AC55 cantilevers. Measurements are made in the middle of a membrane with diameter $2.5 \mu \mathrm{m}$. We show results from two different membranes from the same batch, measured with two different cantilevers. (a) The conservative force quadrature $F_{I}(A)$ and (b) the dissipative quadrature $F_{Q}(A)$. The curves are offset vertically for clarity, with dashed lines corresponding to zero force. (c),(d) The simulated motion of the tip $z(t)$ (blue) and surface $z_{S}(t)$ (orange) for the AC55 cantilever (c),(d) and for the Tap300 cantilever (e),(f). The enlargements in (c),(e) show details of the surface motion around the time marked by the vertical dotted line in (d),(f), respectively. The best-fit parameters used in the simulation are given in the table.

If we start from first principles, even very idealized models would involve many parameters: tip radius and side-wall angle - assuming an axial-symmetric tip; elastic modulus, Poisson ratio, and viscosity — assuming a homogeneous half-space; surface energies and local curvature of the unperturbed surface. All parameters not independently determined should be treated as free when one is fitting AFM data. The fit procedure here uses the multifrequency ImAFM data at some 40 frequencies near resonance. While the quadrature data ( 80 values) do have a different weight (they are measured with a different signal-to-noise ratio), each represents an independent observable with information about the tip-sample interaction. In stark contrast to other quantitative methods in AFM, the large number of observables in relation to the number of free model parameters makes physical interpretation of the fitted parameter values meaningful.

Examining Table I, we recall that the cantilever parameters $f_{0}, Q$, and $k$ are independently calibrated (see Appendix A) and the working distance $w$ can be determined by inspection (amplitude at the initial sharp rise in $\left.F_{I}\right)$. Thus, the five parameters $F_{\text {ad }}, k_{s}, \eta_{s}, k_{v}$, and $\eta_{v}$, are free parameters. We expect that all of these will depend on the detailed shape of the tip. It is therefore not so surprising that Table I shows considerable variation of these parameters between the different probes. We expect that the relaxation time $\tau_{s}=\eta_{s} / k_{s}$ is independent of tip shape as it involves the free relaxation of the sample. Furthermore, $\tau_{v}=\eta_{v} / k_{v}$ might, to first order, be sensitive to tip shape as a blunt tip would result in both larger viscous force and larger elastic force in comparison with a sharper tip. Indeed, we do observe little variation in the values of $\tau_{s}$ and $\tau_{v}$ for the two cantilevers with lower frequency, but there is a reduction of both $\tau_{s}$ and $\tau_{v}$ for the highest-frequency cantilever.

It is interesting to note in Table I and in the table in Fig. 7 that the ratio of time constants

$$
R=\frac{\tau_{s}}{\tau_{v}}=\frac{\eta_{s} k_{v}}{k_{s} \eta_{v}}
$$

is apparently independent of the AFM probe used in the experiment. We call $R$ the "impact-rheology factor" [41], a dimensionless number formed from all four force constants of our PWL viscoelastic model. Our measurements suggests that this factor may be a good quantity for physical characterization of surface viscoelasticity.

To quantify measurement uncertainty in $R$, one should study how various sources of noise in the experiment propagate to the model parameters in the fitting procedure. Further experimental studies and more detailed model analysis are required to study uncertainties and verify if the impact-rheology factor is indeed a reliable measurement of an intrinsic property of the sample interface. 
While this moving-surface model is minimal with regard to the number of parameters, it nevertheless has very complicated dynamics. General statements about its behavior in different parameter regimes are therefore difficult to formulate. The impact-rheology factor represents the relative strength of two viscoelastic processes: Large $R$ means that the sample's free relaxation is slow in comparison with the time needed for the penetrating tip to locally deform the sample. Conversely, small $R$ means that tip penetration is slow in comparison with free relaxation. However, the magnitude of $R$ alone is insufficient to predict the shape of the force-quadrature curves, which also depend on the adhesion force, working distance, and frequency of oscillation. Hysteresis is associated with $\omega_{0} \tau_{s} \gg 1$, large surface lifting with small $k_{s}$ or large $\delta_{0}$, and deep penetration with small $k_{v}$.

\section{CONCLUSIONS}

We presented a minimal model for dynamic atomic force microscopy on viscoelastic materials, accounting for both surface forces and bulk forces. The model is simple in that it is linear where possible, but its dynamics is complex due to the nonlinearity describing the sudden impact and release of the oscillating tip and surface. Unlike traditional nanomechanical analysis of atomic force microscopy, our model takes into account the viscoelastic dynamics of both the penetrating tip and the free sample. The sample dynamics is shown to be quite significant on soft materials, where simulations revealed large-amplitude surface motion. We validated the model by showing excellent agreement with experimental data for a variety of samples. By fitting the model to the data, we extracted viscous and elastic force constants for the surface and bulk. Our analysis indicated that the impact-rheology factor, formed from the dimensionless ratio of these constants, is independent of tip shape and cantilever resonance frequency. This simple model describes numerous measured force-quadrature curves with complex and differing shape, instilling confidence that it captures the essential physics of dynamic atomic force microscopy on soft materials.

\section{ACKNOWLEDGMENTS}

We acknowledge S. Borysov, C. A. van Edysen, J. Wettlaufer, and Xiao-min Lin for helpful discussions, and J. Jureller for help with the measurements. P.-A.T, R.B., D.F., I.D., P.M.C., and D.B.H are grateful for financial support from the Swedish Research Council, the Olle Engkvist Foundation, and the Knut and Allice Wallenberg Foundation. Research in Mons was supported by the European Commission and Wallonia FEDER program, the Science Policy Office of the Belgian Federal Government (BELSPO-PAI VII/5), and the FRS-FNRS PDR project ECOSTOFLEX. Y.W. and H.M.J. acknowledge support from the Office of Naval Research through Grant
No. N00014-17-1-2342 and through the Chicago Materials Research Science and Engineering Center under NSF Grant No. DMR-1420709.

\section{APPENDIX A: CALIBRATION}

Cantilever parameters are determined by the noninvasive thermal calibration method described by Higgins et al. [42], which combines the fluctuation dissipation theorem with Sader's method based on analysis of hydrodynamic damping [43]. With this approach one can extract the three parameters of the cantilever transfer function, $k, f_{0}$, and $Q$, as well as the inverse responsivity of the optical detector used to measure cantilever deflection $\alpha^{-1}(\mathrm{~nm} / \mathrm{V})$, all from one measurement of the thermal Brownian motion of the cantilever near resonance. The method is encapsulated in the recently launched Global Calibration Initiative (GCI) [44], where a thermal noise measurement of $f_{0}$ and $Q$ can be used to get $k$, on the basis of a single hydrodynamic constant determined from averaging over the measurements of many users on the same type of cantilever. We take our measured $f_{0}$ and $Q$ and use the GCI to determine $k_{\text {Sader }}$, allowing us to then determine $\alpha^{-1}$. Note that error in the calibration of $k$ and $\alpha^{-1}$ results in a rescaling of the force axis and the amplitude axis, respectively, which does not change the general shape of the force-quadrature curves.

\section{APPENDIX B: MEASUREMENT DETAILS}

Each measurement (one pixel of the scan) requires $2 \mathrm{~ms}$, corresponding to a frequency spacing in the comb of intermodulation products of $500 \mathrm{~Hz}$, also the measurement bandwidth. To enhance the signal-to-noise ratio, we average the measured intermodulation spectra over several neighboring pixels that show the same type of response, thereby using spatial correlation to reduce the noise. This averaging results in smoother experimental force-quadrature curves without the need to reduce the scan speed.

When the cantilever is oscillating above a surface we often observe significant background forces, not resulting from tip-sample interaction but rather acting over the entire cantilever body. The origin of these background forces might be, for example, a long-range electrostatic force or hydrodynamic squeeze-film damping. To deduce the tip-sample force we need to remove this background interaction. The procedure we use for removing any linear background force is described in Ref. [45].

\section{APPENDIX C: NUMERICAL INTEGRATION AND OPTIMIZATION}

For numerical simulation of the tip and surface motion it is convenient to introduce a nondimensional time $u=$ $\omega_{0} t$, where $f_{0}=\omega_{0} / 2 \pi$ is the resonant frequency of the cantilever in hertz. With this scaling the relaxation times 
become dimensionless parameters, $u_{s}=\omega_{0} \tau_{s}$ and $u_{v}=$ $\omega_{0} \tau_{v}$. Equations (7a) and (7b) then describe a threedimensional dynamic system with state variables $d, d^{\prime}$, and $d_{s}$. For the case $s<0$ this system reads

$$
\begin{gathered}
\frac{d}{d u} d=d^{\prime}, \\
\frac{d}{d u} d^{\prime}=-\frac{1}{Q} d^{\prime}-d+\frac{F_{\mathrm{drive}}}{k}-\frac{F_{\mathrm{ad}}}{k} \\
-\frac{k_{v}}{k}\left(s+u_{v} s^{\prime}\right), \\
\frac{d}{d u} d_{s}=d_{s}^{\prime}=-\frac{d_{s}}{u_{s}}+\frac{k_{v}}{u_{s} k_{s}} \\
\times\left[\frac{F_{\mathrm{ad}}}{k_{v}}+s+u_{v}\left(d^{\prime}-d_{s}^{\prime}\right)\right] .
\end{gathered}
$$

Since the right-hand side of Eq. $(\mathrm{C} 1 \mathrm{~b})$ depends on $s^{\prime}=$ $d^{\prime}-d_{s}^{\prime}$ at each time step, we must first solve Eq. (C1c) for $d_{s}^{\prime}$ and then substitute it into the right-hand side of Eq. (C1b). In terms of the impact-rheology factor $R=\tau_{s} / \tau_{v}$ and the stiffness ratio $K=k_{s} / k_{v}$, we can write Eq. (C1c) as

$$
\frac{d}{d u} d_{s}=\frac{1}{1+R K}\left[-\frac{d_{s}}{u_{s}}+\frac{1}{u_{s} K} \times\left(\frac{F_{\mathrm{ad}}}{k_{v}}+s+u_{v} d^{\prime}\right)\right] .
$$

The prefactor $1 /(1+R K)$ in front of Eq. (C2) describes the strength of the dynamic coupling between the tip and the surface, giving a measure of how much surface motion one can expect.

Numerical integration of the model is performed with the package CVODE, part of the SUNDIALS suite of nonlinear solvers [46]. This integrator has adaptive time stepping. Because the dynamic system is coded in $\mathrm{C}$, the simulation time is reduced by a factor of 100 in comparison with coding in higher-level languages such as MATLAB or PYTHON. This speedup is of great importance as many integrations are required during iteration to find the optimal parameters. For the numerical optimization, the initial conditions for $d$ and $d^{\prime}$ are reset to their measured values for each iteration, whereas the initial condition for $d_{s}$ is set to zero on the first iteration, and it is then estimated from the previous integration for all subsequent iterations.

We use the PYTHON library scipy.optimize.leastsq, which is a wrapper for MINPACK's implementation of the Levenberg-Marquardt algorithm. The algorithm performs a least-squares minimization of the array of residuals $r=$ $\left\{\operatorname{Re}\left(\hat{F}_{\text {expt }}-\hat{F}_{\text {sim }}\right), \operatorname{Im}\left(\hat{F}_{\text {expt }}-\hat{F}_{\text {sim }}\right)\right\}$, where the $\hat{F}(\omega)$ are experimental and simulated complex force amplitudes at
40 frequencies. The method finds a local minimum, starting from good initial parameter values determined by trial and error.

\section{APPENDIX D: CHANGING THE WORKING DISTANCE}

Figure 8 shows the dynamic-force-quadrature curves measured with the NSC15 and AC55 cantilevers. The measured curves are taken at different set points. The simulated curves for each cantilever have different working distance, with all other model parameters being the same.

(a)
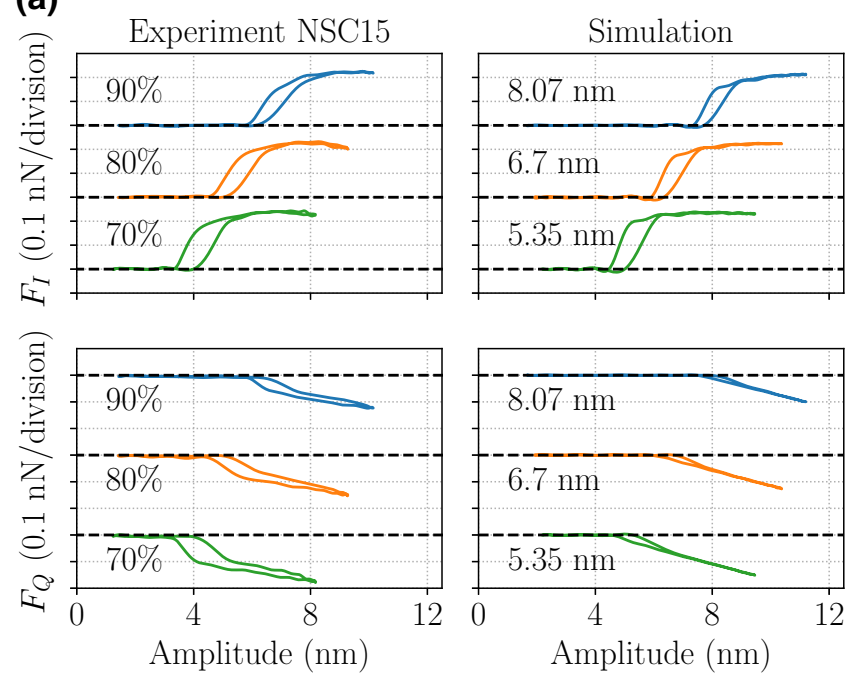

(b)
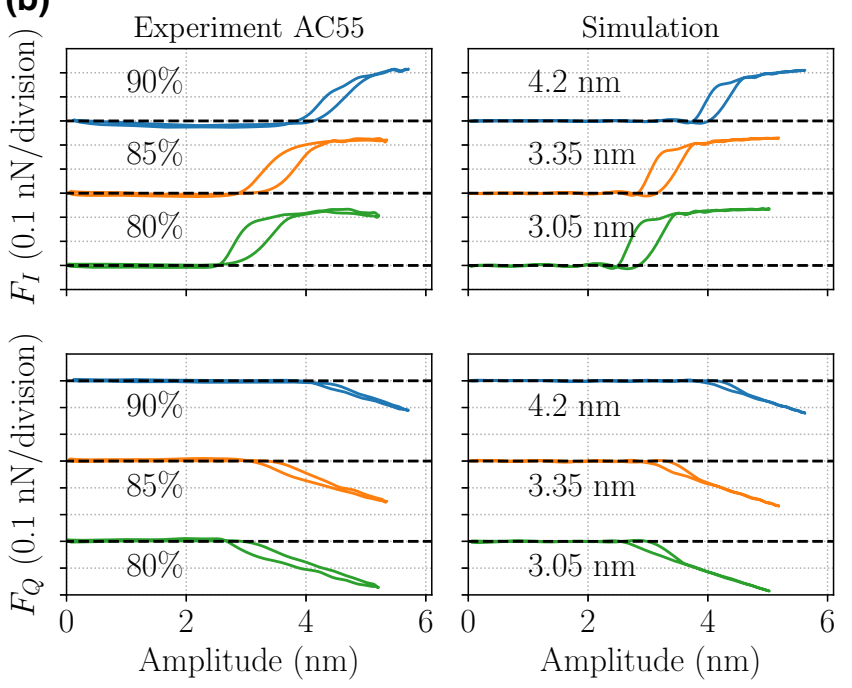

FIG. 8. Plots equivalent to those in Fig. 6 for the NSC15 cantilever (top) and the AC55 cantilever (bottom).

[1] A. Yango, J. Schape, C. Rianna, H. Doschke, and M. Radmacher, Measuring the viscoelastic creep of soft samples by step response AFM, Soft Matter 12, 8297 (2016). 
[2] Y. M. Efremov, W.-H. Wang, S. D. Hardy, R. L. Geahlen, and A. Raman, Measuring nanoscale viscoelastic parameters of cells directly from AFM force-displacement curves, Sci. Rep. 7, 1541 (2017).

[3] F. M. Hecht, J. Rheinlaender, N. Schierbaum, W. H. Goldmann, B. Fabry, and T. E. Schaffer, Imaging viscoelastic properties of live cells by AFM: power-law rheology on the nanoscale, Soft Matter 11, 4584 (2015).

[4] B. K. Connizzo and A. J. Grodzinsky, Tendon exhibits complex poroelastic behavior at the nanoscale as revealed by high-frequency AFM-based rheology, J. Biomech. 54, 11 (2017).

[5] D. C. Hurley and J. P. Killgore, Dynamic Contact AFM Methods for Nanomechanical Properties (John Wiley \& Sons, Inc., Hoboken, New Jersey, 2013), p. 115.

[6] G. Stan and R. S Gates, Intermittent contact resonance atomic force microscopy, Nanotechnology 25, 245702 (2014).

[7] I. Chakraborty and D. G. Yablon, Temperature dependent loss tangent measurement of polymers with contact resonance atomic force microscopy, Polymer 55, 1609 (2014).

[8] M. Chyasnavichyus, S. L. Young, and V. V. Tsukruk, Recent advances in micromechanical characterization of polymer, biomaterial, and cell surfaces with atomic force microscopy, Jpn. J. Appl. Phys. 54(8S2), 08LA02 (2015).

[9] H. K. Nguyen, M. Ito, and K. Nakajima, Elastic and viscoelastic characterization of inhomogeneous polymers by bimodal atomic force microscopy, Jpn. J. Appl. Phys. 55(8S1), 08NB06 (2016).

[10] E. Rezaei and J. A. Turner, Contact resonance AFM to quantify the in-plane and out-of-plane loss tangents of polymers simultaneously, Appl. Phys. Lett. 110, 101902 (2017).

[11] P. M. Claesson, I. Dobryden, G. Li, Y. He, H. Huang, P.-A. Thorén, and D. B. Haviland, From force curves to surface nanomechanical properties, Phys. Chem. Chem. Phys. 19, 23642 (2017).

[12] A. B. Churnside, R. C. Tung, and J. P. Killgore, Quantitative contact resonance force microscopy for viscoelastic measurement of soft materials at the solid-liquid interface, Langmuir 31, 11143 (2015).

[13] A. Maali, R. Boisgard, H. Chraibi, Z. Zhang, H. Kellay, and A. Würger, Viscoelastic Drag Forces and Crossover from No-Slip to Slip Boundary Conditions for Flow Near Air-Water Interfaces, Phys. Rev. Lett. 118, 084501 (2017).

[14] H. Schlicke, E. W. Leib, A. Petrov, J. H. Schröder, and T. Vossmeyer, Elastic and viscoelastic properties of crosslinked gold nanoparticles probed by AFM bulge tests, J. Phys. Chem. C 118, 4386 (2014).

[15] T. Ando, T. Uchihashi, and N. Kodera, High-speed AFM and applications to biomolecular systems, Annu. Rev. Biophys. 42, 393 (2013).

[16] B. P. Brown, L. Picco, M. J. Miles, and C. F. J. Faul, Opportunities in high-speed atomic force microscopy, Small $\mathbf{9}$, 3201 (2013)

[17] C. A. Amo, A. P. Perrino, A. F. Payam, and R. Garcia, Mapping elastic properties of heterogeneous materials in liquid with angstrom-scale resolution, ACS Nano 11, 8650 (2017).
[18] A. X. Cartagena-Rivera, W.-H. Wang, R. L. Geahlen, and A. Raman, Fast, multi-frequency, and quantitative nanomechanical mapping of live cells using the atomic force microscope, Sci. Rep. 5, 11692 (2015).

[19] F. Peterka, Dynamics of the Impact Oscillator (Springer, Dordrecht, 1999), p. 283.

[20] D. Platz, E. A. Tholen, D. Pesen, and D. B. Haviland, Intermodulation atomic force microscopy, Appl. Phys. Lett. 92, 153106 (2008).

[21] R. Miller, J. K. Ferri, A. Javadi, J. Krägel, N. Mucic, and R. Wüstneck, Rheology of interfacial layers, Colloid Polym. Sci. 288, 937 (2010).

[22] H. Hertz, On the contact of elastic solids, J. Reine Angew. Math. 92, 156 (1881).

[23] A. Labuda, M. Kocun, W. Meinhold, D. Walters, and R. Proksch, Generalized Hertz model for bimodal nanomechanical mapping, Beilstein J. Nanotechnol. 7, 970 (2016).

[24] A. He and J. S. Wettlaufer, Hertz beyond belief, Soft Matter 10, 2264 (2014).

[25] D. Platz, D. Forchheimer, E. A. Tholén, and D. B. Haviland, The role of nonlinear dynamics in quantitative atomic force microscopy, Nanotechnology 23, 265705 (2012).

[26] S. D. Solares, Nanoscale effects in the characterization of viscoelastic materials with atomic force microscopy: coupling of a quasi-three-dimensional standard linear solid model with in-plane surface interactions, Beilstein J. Nanotechnol. 7, 554 (2016).

[27] P. Attard, Measurement and interpretation of elastic and viscoelastic properties with the atomic force microscope, J. Phys. Condens. Matter 19, 473201 (2007).

[28] K. L. Johnson, K. Kendall, and A. D. Roberts, Surface energy and the contact of elastic solids, Proc. R. Soc. Lond. A Math. Phys. Sci. 324, 301 (1971).

[29] R. W. Style, C. Hyland, R. Boltyanskiy, J. S. Wettlaufer, and E. R. Dufresne, Surface tension and contact with soft elastic solids, Nat. Commun. 4, 2728 (2013).

[30] H.-J. Butt, J. T. Pham, and M. Kappl, Forces between a stiff and a soft surface, Curr. Opin. Colloid Interface Sci. 27, 82 (2017).

[31] J. Melcher, S. Hu, and A. Raman, Invited article: Veda: a web-based virtual environment for dynamic atomic force microscopy, Rev. Sci. Instrum. 79, 061301 (2008).

[32] J. H. Cantrell and S. A. Cantrell, Cantilever Dynamics: Theoretical Modeling (Springer, Berlin, 2013), p. 47.

[33] D. B. Haviland, C. A. van Eysden, D. Forchheimer, D. Platz, H. G. Kassa, and P. Leclere, Probing viscoelastic response of soft material surfaces at the nanoscale, Soft Matter 12, 619 (2016).

[34] D. Platz, D. Forchheimer, E. A. Tholén, and D. B. Haviland, Interaction imaging with amplitude-dependence force spectroscopy, Nat. Commun. 4, 1360 (2013).

[35] D. B. Haviland, Quantitative force microscopy from a dynamic point of view, Curr. Opin. Colloid Interface Sci. 27, 74 (2017).

[36] H. Huang, I. Dobryden, P.-A. Thorén, L. Ejenstam, J. Pan, M. L. Fielden,D. B. Haviland, and P. M. Claesson, Local surface mechanical properties of PDMS-silica nanocomposite probed with intermodulation AFM, Compos. Sci. Technol. 150, 111 (2017). 
[37] HarmoniX test sample, Bruker Corp.

[38] J. He, P. Kanjanaboos, N. Laszlo Frazer, A. Weis, X.-M. Lin, and H. M. Jaeger, Fabrication and mechanical properties of large-scale freestanding nanoparticle membranes, Small 6, 1449 (2010).

[39] P. Kanjanaboos, X.-M. Lin, J. E. Sader, S. M. Rupich, H. M. Jaeger, and J. R. Guest, Self-assembled nanoparticle drumhead resonators, Nano Lett. 13, 2158 (2013).

[40] Y. Wang, H. Chan, B. Narayanan, S. P. McBride, S. K. R. S. Sankaranarayanan, X.-M. Lin, and H. M. Jaeger, Thermomechanical response of self-assembled nanoparticle membranes, ACS Nano 11, 8026 (2017).

[41] Not to be confused with the journal Rheology, which has an impact factor.

[42] M. J. Higgins, R. Proksch, J. E. Sader, M. Polcik, S. Mc. Endoo, J. P. Cleveland, and S. P. Jarvis, Noninvasive determination of optical lever sensitivity in atomic force microscopy, Rev. Sci. Instrum. 77, 013701 (2006).
[43] J. E. Sader, J. A. Sanelli, B. D. Adamson, J. P. Monty, X. Wei, S. A. Crawford, J. R. Friend, I. Marusic, P. Mulvaney, and E. J. Bieske, Spring constant calibration of atomic force microscope cantilevers of arbitrary shape, Rev. Sci. Instrum. 83, 103705 (2012).

[44] J. E. Sader, R. Borgani, C. T. Gibson, D. B. Haviland, M. J. Higgins, J. I. Kilpatrick, J. Lu, P. Mulvaney, C. J. Shearer, A. D. Slattery, P.-A. Thorén, J. Tran, H. Zhang, H. Zhang, and T. Zheng, A virtual instrument to standardise the calibration of atomic force microscope cantilevers, Rev. Sci. Instrum. 87, 093711 (2016).

[45] R. Borgani, P.-A. Thorén, D. Forchheimer, I. Dobryden, S. M. Sah, P. M. Claesson, and D. B. Haviland, Background-Force Compensation in Dynamic Atomic Force Microscopy, Phys. Rev. Appl. 7, 064018 (2017).

[46] A. C. Hindmarsh, P. N. Brown, K. E. Grant, S. L. Lee, R. Serban, D. E. Shumaker, and C. S. Woodward, Sundials: suite of nonlinear and differential/algebraic equation solvers, ACM Trans. Math. Softw. 31, 363 (2005). 